\title{
Association of Single-Nucleotide Polymorphisms in ABCC5 Gene with Primary Angle Closure Glaucoma and the Ocular Biometric Parameters in a Northern Chinese Population
}

\author{
Shaolin Wang ${ }^{\mathrm{a}}$ Wenjuan Zhuang ${ }^{\mathrm{a}}$ Wen Zhang ${ }^{\mathrm{a}}$ Manyun $\mathrm{Xu}^{\mathrm{a}}$ Wenjing $\mathrm{Li}^{\mathrm{b}}$ \\ Shunyu Piao ${ }^{a}$ Zhongqi Xue ${ }^{c}$ Bo Cai $^{b}$ Shaoping Ha ${ }^{a}$ \\ aDepartment of Ophthalmology, Ning Xia Eye Hospital, People's Hospital of Ningxia Hui Autonomous Region \\ (First Affiliated Hospital of Northwest University for Nationalities, Ningxia Clinical Research Center on Diseases \\ of Blindness in Eye), Yinchuan, China; ${ }^{b}$ Clinical Medical College of Ningxia Medical University, Yinchuan, China; \\ 'Qindao Binhai University Affiliated Hospital, Qindao, China
}

\section{Keywords}

$A B C C 5 \cdot \mathrm{PACG} \cdot \mathrm{ACD} \cdot \mathrm{AL} \cdot$ Single-nucleotide polymorphisms

\begin{abstract}
Background: The rs1401999 gene in $A B C C 5$ gene was the first locus confirmed by a genome-wide association study (GWAS) to be associated with both anterior chamber depth (ACD) and primary angle closure glaucoma (PACG); however, this locus was of obvious heterogeneity among different populations in the GWAS, and the conclusion has not been further verified by other studies. Therefore, this study was carried out to investigate whether the single-nucleotide polymorphisms (SNPs) in $A B C C 5$ gene are associated with PACG and the ocular biometric parameters ACD and axial length (AL) in samples from northern China. Methods: Casecontrol association study included 500 PACG patients and 720 unrelated controls from northern China, and genotyping was performed for ten SNPs in $A B C C 5$ gene using an improved multiplex ligation detection reaction technique. The association between these SNPs and risk of PACG was estimated by PLINK using a logistic regression model, while the association between genotypes and ocular biometric parameters was performed by SPSS using generalized estima-
\end{abstract}

tion equation. Results: An SNP rs4148568 $(p=0.046)$ and a haplotype TCGGAG $(p=0.0364)$ in $A B C C 5$ were associated with PACG, and rs 4148568 was nominally associated with $A L$ $(\beta=0.092, p=0.08)$. Conclusions: The SNP rs4148568 and a haplotype TCGGAG in ABCC5 contribute to PACG in northern Chinese people. In addition, rs 4148568 might be associated with the $A L$, the variant allele of which may have effect of making the AL longer. Further studies are needed to elucidate the exact mechanism of $A B C C 5$ in the progress of PACG.

(c) 2020 The Author(s)

Published by S. Karger AG, Basel

\section{Introduction}

Primary angle closure glaucoma (PACG) is a major subtype of glaucoma in Asia and also the most common cause of bilateral glaucoma blindness worldwide $[1,2]$. PACG is a multifactorial disease and has been proven to have a strong genetic basis. To date, two genome-wide association studies (GWASs) on PACG have been conducted, and 8 genetic loci showed strong associations with

Shaolin Wang and Wenjuan Zhuang contributed equally to this publication.
(C) 2020 The Author(s)

Published by S. Karger AG, Basel

This is an Open Access article licensed under the Creative Commons Attribution-NonCommercial-4.0 International License (CC BY-NC) (http://www.karger.com/Services/OpenAccessLicense), applicable to the online version of the article only. Usage and distribution for commercial purposes requires written permission.
Correspondence to:

Wenjuan Zhuang, zh_wenj@163.com 
PACG [3, 4]: rs11024102 in PLEKHA7, rs3753841 in COL11A1, rs1015213 located between PCMTD1 and ST18, rs3816415 in EPDR1, rs1258267 in CHAT, rs736893 in GLIS3, rs7494379 in FERMT2, and rs3739821 mapping between DPM2 and FAM102A. Besides, another GWAS on anterior chamber depth (ACD), a locus, rs1401999 in the $A B C C 5$ gene, was also found to be associated with PACG [5]. These discoveries based on the GWAS are reliable since they were replicated in a large sample in the replication stage; however, the conclusions were inconsistent from those of other researchers who have validated the results of GWAS by Vithana et al. [3] in different cohorts and different regions [6-8].

Beyond that, some researchers tried to find out whether the 3 susceptibility loci identified by the first GWAS on PACG were associated with ocular biometric parameters [9-11], among which Day et al. [9] confirmed an association of the locus rs 1015213 with ACD in a European cohort [9], while no association was found in other studies $[10,11]$. Validation in additional independent cohorts and analysis of the relationship between these genes and ocular biometric parameters will help to determine the contributions of these genes in the development of PACG. In our previous study, we have evaluated the association of the 8 susceptibility loci which were reported by the GWAS with PACG as well as ocular biometric parameters ACD and axial length (AL), and no correlation was found between these loci and ocular biometric parameters [12]. The rs1401999 in ABCC5 gene was the first locus confirmed by the GWAS to be associated with both ACD and PACG; however, this locus was of obvious heterogeneity among different populations in the GWAS, and the conclusion has not been further verified by other studies. Therefore, this case-control study was designed to investigate whether the gene variants in $A B C C 5$ gene are associated with PACG and the ocular biometric parameters $\mathrm{ACD}$ and $\mathrm{AL}$ in a northern Chinese cohort.

\section{Materials and Methods}

\section{Subjects}

This study was conducted in accordance with the tenets of the Declaration of Helsinki; ethical approval was obtained from the Ningxia People's Hospital; and written informed consent was obtained from each individual prior to the study. A total of 1,220 unrelated Chinese individuals were recruited from the northern regions of China, including 500 PACG patients and 720 unaffected controls. The detailed ophthalmic examinations for every participant, and the inclusion and exclusion criteria as well as the protocol of genetic DNA extraction were identical to those in our previous study [13].

Association of $A B C C 5$ with PACG, and $\mathrm{ACD}$ and $\mathrm{AL}$
Table 1. Demographic features of study participants

\begin{tabular}{llcl}
\hline & Cases & Controls & $p$ value \\
\hline$n$ & 500 & 720 & \\
Age (mean \pm SD), years & $63.77 \pm 9.576$ & $71.82 \pm 7.2$ & $0.000^{\#}$ \\
$\begin{array}{l}\text { Sex, } n(\%) \\
\quad \text { Male }\end{array}$ & $147(29.4)$ & $332(46.1)$ & \\
$\quad$ Female & $353(70.6)$ & $388(53.9)$ & $0.000^{*}$ \\
Nationality, $n(\%)$ & & & \\
$\quad$ Han & & & \\
$\quad$ Hui & $407(81.4)$ & $591(82.1)$ & $0.761^{*}$ \\
\hline
\end{tabular}

\# The $p$ value was tested by $t$ test. * The $p$ value was assessed by $\chi^{2}$ test.

\section{SNP Selection and Genotyping}

A total of ten tag single-nucleotide polymorphisms (SNPs) in ABCC5 gene were selected from HapMap Beijing Han Chinese population (International HapMap Project) utilizing the tagger program implemented in Haploview 4.2 software (Daly Lab at the Broad Institute, Cambridge, MA) via a pairwise tagging algorithm; there were rs112754234, rs9838667, rs1016752, rs4912517, rs1401999, rs6767306, rs7620781, rs6773408, rs4148568, and rs1879258. Each tag SNP had to adhere to the following criteria: $r^{2}$ threshold $>0.8$ and minimal allele frequency $>10 \%$. SNP genotyping was conducted by the Genesky Biotechnologies Inc (Shanghai, China) using an improved multiplex ligation detection reaction technique.

\section{Statistical Analysis}

The comparisons of demographic characteristics between cases and controls and the correlations analyses between genotypes and ocular biometric parameters were implemented by SPSS software (version 17.5: SPSS Science, Chicago, IL, USA); the genetic association analyses were performed by PLINK (version 1.07; http:// pngu.mgh.harvard.edu/ purcell/plink/, in the public domain); the detailed statistical analysis methods were the same as our previous study [13]. The statistical power was evaluated by the Power and Sample Size Calculation (PS; version 3.1.232).

\section{Results}

This study included 500 PACG patients and 720 control subjects, as shown in Table 1; there were no significant differences in ethnicity between cases and controls. However, the cases were significantly younger (mean age $63.77 \pm 9.58$ years vs. $71.82 \pm 7.2$ years; $p=0.000)$ and included more women ( 70.6 vs. $53.9 \%$; $p=0.000$ ) than the control group.

Genotype frequencies of all SNPs were within the Hardy-Weinberg equilibrium (HWE) $(p>0.05)$, with the exception of rs9838667 (Table 2), which was slightly deviant in the case group $(p=0.045)$. Among the 10 SNPs, sig- 


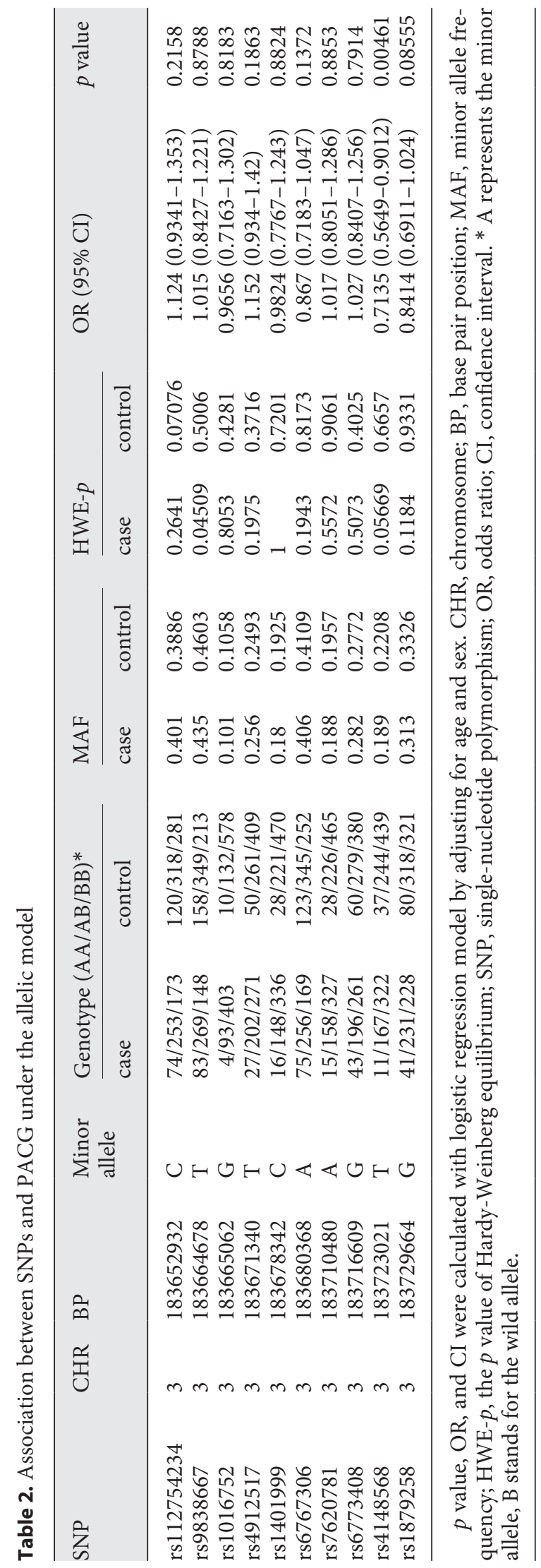

nificant genetic association with PACG was identified for rs4148568 after correction for age and sex using logistic regression (Table 2 ) and the association remained significant after Bonferroni correction $(p=0.046)$. In linkage disequilibriumanalysis, rs9838667, rs1016752, rs4912517, rs1401999, rs6767306, and rs7620781 were found to be in one linkage disequilibrium block (Fig. 1), and a haplotype TCGGAG showed a significant association with PACG ( $p=0.00567)$; after 10,000 permutations, the association still survived ( $p=0.0364$; Table 3 ).

Since the recruited participants included two ethnicities, we also performed a subanalysis within the Hui and Han groups. After correction for age and sex using logistic regression, rs4148568 was found to be associated with PACG in the Han cohort ( $p=0.009858)$, while rs4912517 was associated with PACG in the Hui cohort $(p=0.02587)$ (Table 4). However, the significances were lost after the Bonferroni correction. A meta-analysis of the two different ethnicities was then performed, in which rs4148568 still showed significant association with PACG, and the meta-analysis $p$ value ( $p=0.004606)$ was almost the same as that in the initial overall analysis (Table 4 ). In addition, rs4912517 was found to be of significant heterogeneity between Hui and Han groups $\left(I^{2}=71.28 \%\right.$, $p$ value of $Q$ test $=0.0621)$ (Table 4$)$.

In the association testing between the ten SNP genotypes and ocular biometric parameters AL and ACD using generalized estimation equation (GEE) tests, we found rs4148568 was associated with the $\mathrm{AL}(p=0.008)$, the variant allele of which may have effect of making the AL longer $(\beta=0.092)$ (Table 5); however, this association was lost after the Bonferroni correction $(p=0.08)$.

The powers of this study for these ten SNPs are therefore different because of the differences of their minor allele frequencies. Assuming an allelic odds ratio of 1.29 (derived from the GWAS by Nongpiur et al. [5], the odds ratio of rs1401999 in Beijing Chinese cohort), our sample size provides more than $75 \%$ of statistical power to detect a significant association at the a level of 0.05 with the exception of rs1016752, of which the statistical power is $53.9 \%$.

\section{Discussion}

We explored the associations of tag SNPs in ABCC5 gene with PACG and the ocular biometric parameters $\mathrm{ACD}$ and $\mathrm{AL}$ in a northern Chinese population in our study. The SNP rs4148568 and a haplotype TCGGAG were associated with PACG significantly, and the variant 
Fig. 1. Six SNPs are presented to the one haplotype block in HapMap CHB population, which were determined by the Haploview 4.2 program. Darker shades suggest higher linkage disequilibrium. SNPs, single-nucleotide polymorphism.

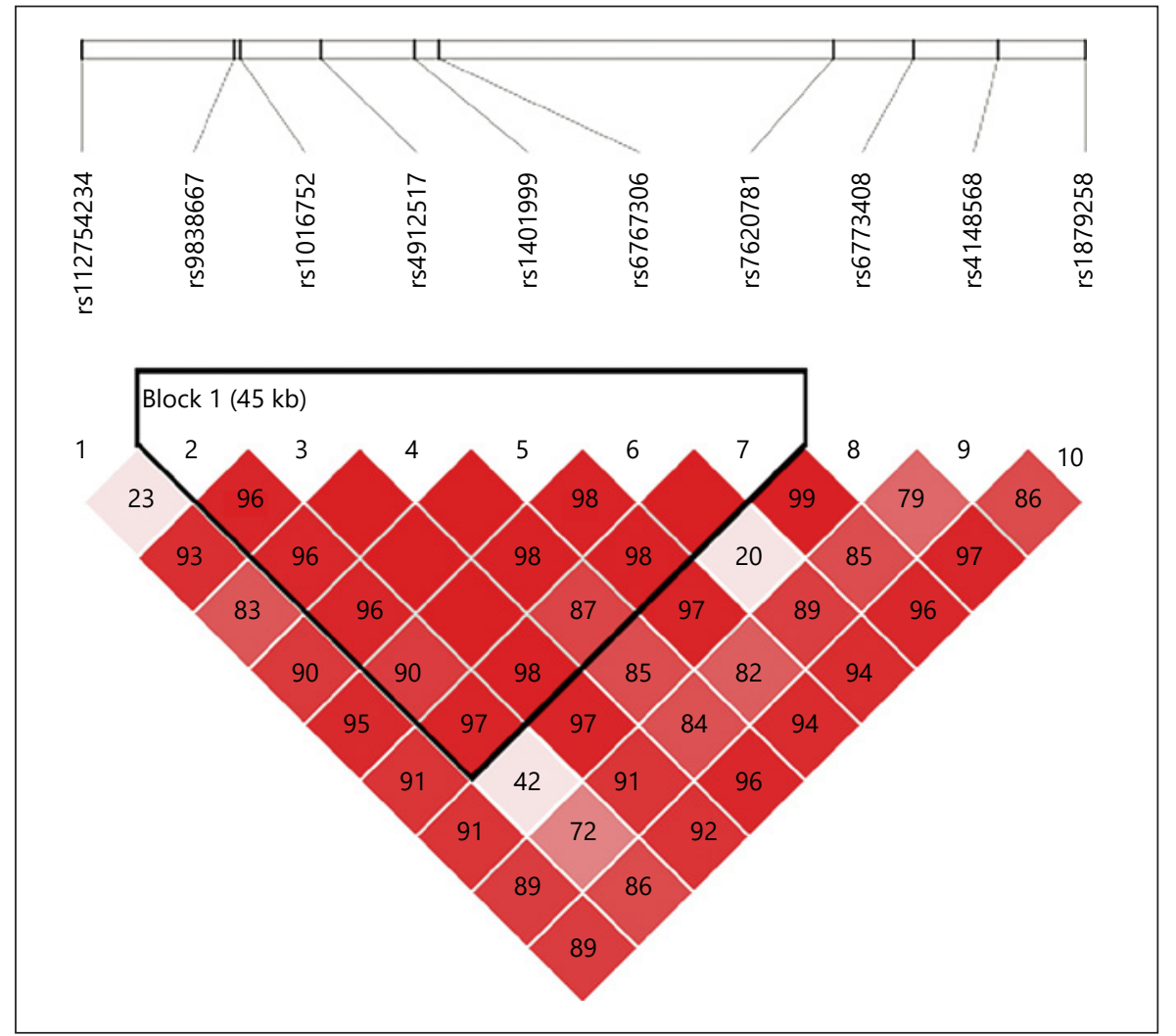

Table 3. Haplotype frequencies in PACG and control cohorts

\begin{tabular}{|c|c|c|c|c|c|c|}
\hline SNP & Haplotype & Freq of cases $(\%)$ & Freq of controls (\%) & $\mathrm{OR}^{\#}$ & $p$ value ${ }^{\#}$ & $p$ permutation $*$ \\
\hline rs9838667, rs1016752, rs4912517, & TGGCGA & 0.09962 & 0.1019 & 0.99 & 0.949 & 1 \\
\hline \multirow[t]{5}{*}{ rs1401999, rs6767306, rs7620781 } & TCGCGA & 0.078 & 0.08422 & 1.03 & 0.86 & 1 \\
\hline & TCGGAG & 0.00497 & 0.02335 & 0.241 & 0.00567 & 0.0364 \\
\hline & GCGGAG & 0.4009 & 0.3852 & 0.945 & 0.558 & 0.993 \\
\hline & TCTGGG & 0.2413 & 0.2382 & 1.12 & 0.309 & 0.8881 \\
\hline & GCGGGG & 0.156 & 0.1481 & 1.06 & 0.645 & 0.998 \\
\hline
\end{tabular}

PACG, primary angle closure glaucoma; SNP, single-nucleotide polymorphism; OR, odds ratio. \# OR and $p$ value were calculated with logistic regression model by adjusting for age and sex. ${ }^{*}$ A total of 10,000 permutations were performed.

allele of rs4148568 was simultaneously found to be nominally correlated with a longer AL. ABCC5, known as multidrug-resistant protein 5 (MRP5), has been reported to play a role in tissue defense and cellular signal transduction $[14,15]$. It is expressed in most human tissues, including cornea, ciliary body, lens and retinal pigment epithelial cells, and retina [5, 16, 17]. Nongpiur et al. [5] found that $A B C C 5$ gene was associated with ACD and weakly associated with the AL, and the rs1401999 locus, which is located in the intronic region, was associated with an increase in the risk of PACG. Tang et al. [18] evaluated the association of some exons in $A B C C 5$ and its 3 adjacent and strongly linked genes with PACG in order to find out any coding variants associated with PACG in this region; unfortunately, no significant association was found between the target loci and PACG. Nevertheless, in our study, the notable association between $A B C C 5$ and PACG was indeed confirmed. Interestingly, the original positive association between rs1401999 and PACG was not replicated, while another locus rs4148568 and a haplotype TCGGAG containing the wild allele $G$ of the rs1401999 locus were verified to be associated with PACG. 
Table 4. Association between SNPs and PACG in different ethnicities and results of meta-analysis

\begin{tabular}{|c|c|c|c|c|c|c|c|c|}
\hline \multirow[t]{2}{*}{ SNP } & \multicolumn{2}{|l|}{$p$ value } & \multicolumn{2}{|l|}{ OR (95\% CI) } & \multirow[t]{2}{*}{$p$ meta } & \multirow[t]{2}{*}{ OR-meta } & \multirow[t]{2}{*}{$I^{2}$} & \multirow[t]{2}{*}{$p$ het } \\
\hline & Hui & Han & Hui & Han & & & & \\
\hline rs9838667 & 0.438 & 0.837 & $1.18(0.7771-1.79)$ & $0.9784(0.7944-1.205)$ & 0.8701 & 1.0157 & 0 & 0.4311 \\
\hline rs1016752 & 0.7834 & 0.9614 & $0.9198(0.5067-1.67)$ & $0.9915(0.7012-1.402)$ & 0.8573 & 0.9729 & 0 & 0.831 \\
\hline rs4912517 & 0.02587 & 0.8354 & $1.665(1.063-2.607)$ & $1.026(0.807-1.304)$ & 0.3376 & 1.2575 & 71.28 & 0.0621 \\
\hline rs7620781 & 0.4787 & 0.5208 & $0.8268(0.4886-1.399)$ & $1.09(0.8381-1.417)$ & 0.7957 & 1.0315 & 0 & 0.3569 \\
\hline rs6773408 & 0.3776 & 0.4181 & $0.8192(0.526-1.276)$ & $1.098(0.8758-1.376)$ & 0.748 & 1.0335 & 24.98 & 0.2483 \\
\hline rs4148568 & 0.241 & 0.009858 & $0.7284(0.4289-1.237)$ & $0.7091(0.5461-0.9206)$ & 0.004606 & 0.7128 & 0 & 0.929 \\
\hline rs1879258 & 0.9713 & 0.05553 & $0.9918(0.6323-1.556)$ & $0.8064(0.6469-1.005)$ & 0.08269 & 0.8393 & 0 & 0.4184 \\
\hline
\end{tabular}

OR, odds ratio; CI, confidence interval; $I^{2}$, measures heterogeneity; $p$ het, $p$ value for heterogeneity; $p$ value, OR, and CI were calculated with logistic regression model by adjusting for age and sex. SNPs, single-nucleotide polymorphisms; PACG, primary angle closure glaucoma; OR-meta, OR obtained by meta-analysis; $p$ meta, $p$ value obtained by meta-analysis; if the $I^{2}$ value was $\geq 50 \%$, it takes the value of random-effects; otherwise, a fixed-effects $q$ model was adopted.

Table 5. Association between SNPs, and AL and ACD

\begin{tabular}{|c|c|c|c|c|c|c|c|}
\hline \multirow[t]{2}{*}{ SNP } & \multirow{2}{*}{$\begin{array}{l}\text { Minor } \\
\text { allele }\end{array}$} & \multicolumn{3}{|c|}{$\operatorname{AL}(22.92 \pm 0.891 ; 20.01-25.51)^{*}$} & \multicolumn{3}{|c|}{$\operatorname{ACD}(2.74 \pm 0.474 ; 0.67-4.51)^{*}$} \\
\hline & & $B$ & $95 \%$ CI & $p$ value & $B$ & $95 \%$ CI & $p$ value \\
\hline rs112754234 & $\mathrm{C}$ & 0.015 & $-0.066-0.096$ & 0.714 & 0.022 & $-0.017-0.062$ & 0.267 \\
\hline rs9838667 & $\mathrm{T}$ & -0.033 & $-0.107-0.04$ & 0.373 & 0.001 & $-0.037-0.04$ & 0.944 \\
\hline rs1016752 & G & -0.062 & $-0.187-0.064$ & 0.337 & -0.022 & $-0.083-0.039$ & 0.485 \\
\hline rs4912517 & $\mathrm{T}$ & -0.008 & $-0.096-0.079$ & 0.85 & 0.006 & $-0.037-0.05$ & 0.774 \\
\hline rs1401999 & $\mathrm{C}$ & -0.065 & $-0.159-0.03$ & 0.178 & -0.022 & $-0.072-0.028$ & 0.384 \\
\hline rs6767306 & $\mathrm{A}$ & 0.037 & $-0.044-0.118$ & 0.37 & -0.004 & $-0.045-0.038$ & 0.856 \\
\hline rs7620781 & A & -0.062 & $-0.157-0.034$ & 0.207 & -0.012 & $-0.061-0.037$ & 0.629 \\
\hline rs6773408 & G & -0.061 & $-0.145-0.023$ & 0.156 & -0.019 & $-0.061-0.023$ & 0.379 \\
\hline rs4148568 & $\mathrm{T}$ & 0.092 & $0.024-0.160$ & 0.008 & 0.002 & $-0.048-0.052$ & 0.925 \\
\hline rs1879258 & G & 0.05 & $-0.033-0.132$ & 0.239 & 0.004 & $-0.04-0.049$ & 0.844 \\
\hline
\end{tabular}

$p, p$ value for association, adjusting for age and sex; SNPs, single-nucleotide polymorphisms; AL, axial length; $\mathrm{ACD}$, anterior chamber depth. * The mean $\pm \mathrm{SD}$, and the range of measured values of AL, or ACD; $\beta$, per-allele effect in ACD/AL.

Based on our result, we hold the view that the rs1401999 locus may act in the pathogenesis of PACG through synergistic action with other loci in our cohort since there was no linkage disequilibrium between rs4148568 and rs1401999, while pairwise $r^{2}$ is less than 0.1. Besides that, the obvious heterogeneity at the $A B C C 5$ locus among the different populations (the $I^{2}$ value of which was $60.6 \%$ ) in the GWAS by Nongpiur et al. [5] maybe another consideration not to be neglected, resulting in the inconsistency about the associated locus. Hence, a comprehensive casecontrol association study between $A B C C 5$ and PACG in different populations should be further explored to more completely understand the role of this gene. Similarly, no association was identified between rs1401999 and ACD as well as AL, but rs4148568 was found to be nominally associated with the AL in the present study, and the variant allele of rs4148568 may have effect of making the AL longer. The association of the rs4148568 locus with PACG and its possible role in the regulation of AL will provide new clues to understand the mechanism of $A B C C 5$ gene in PACG. 
In general, binocular biometric parameters can better reflect the genetic characteristics, thus, considering the potential correlation between binocular data, we used GEE in our study since GEE is suitable for statistical analysis of correlated data $[19,20]$ to evaluate the association between genotypes and ocular biometric parameters. Although the association between rs4148568 and the AL was no longer significant after multiple testing corrections, as we know, the Bonferroni correction is known to be conservative for positively correlated $p$ values [21], and the corrected $p$ value was very close to 0.05 in our result. Hence, to some extent, we still infer that the $A B C C 5$ gene plays a role in the pathogenesis of glaucoma by directly or indirectly regulating the AL. Further analysis is still required to identify the veritable correlation and how they contribute to PACG.

In the subgroup analyses within two different ethnic groups, rs4912517 was nominally associated with PACG in the Hui cohort and found to be of significant heterogeneity between Hui and Han groups; in view of that the Hui sample size included in this study was small (93 cases of PACG, 129 controls), the exact association between the rs4912517 locus and PACG in Hui population is worth further exploring. The meta-analysis of the two different ethnic groups was then performed, and the results were consistent with those of the initial overall analysis. This consequence further confirms that the results of our initial analysis are reliable.

Several limitations of our study should be noted. First, the statistical power of our current sample size which is used to evaluate the association within two different ethnic groups is not strong enough. Second, many ocular biometric parameters were closely related to PACG, but in the present study, only the AL and ACD were included to assess the correlation. Therefore, more in-depth study with a large sample size is still necessary in the future.

In summary, the present case-control study validates the association between $A B C C 5$ and PACG; meanwhile, rs4148568 in $A B C C 5$ might be associated with the AL, the variant allele of which may have effect of making the $\mathrm{AL}$ longer. Further research is needed to elucidate the exact mechanism of $A B C C 5$ in the progress of PACG.

\section{Acknowledgements}

The authors thank all the patients and participants.

\section{Statement of Ethics}

This study was approved by the Ethics Committee of the People's Hospital of Ningxia Hui Autonomous Region (No. 2016-031) and met the tenets of the Declaration of Helsinki, and written informed consent was obtained from all of the subjects prior to the study.

\section{Conflict of Interest Statement}

The authors declare that they have no competing interests.

\section{Funding Sources}

This work was supported by grants from the National Natural Science Foundation of China [81460093] and Ningxia Nature Science Funding from the Department of Science and Technology of Ningxia Hui Autonomous Region (NZ16194).

\section{Author Contributions}

S.W. performed clinical examinations, carried out the technical work in the laboratory, wrote the manuscripts, and analyzed the data; W.Z. supervised the overall study, wrote the manuscripts, and analyzed the data; W.Z., M.X., W.L., S.P., Z.X., and B.C. carried out the technical work in the laboratory, including patient's requirements, and reviewed the files for clinical information; S.H. performed clinical examinations and supervised the overall study. All authors read and approved the final manuscript.

\section{References}

1 Tham YC, Li X, Wong TY, Quigley HA, Aung $\mathrm{T}$, Cheng CY. Global prevalence of glaucoma and projections of glaucoma burden through 2040: a systematic review and meta-analysis. Ophthalmology. 2014;121(11):2081-90.

2 Quigley HA, Congdon NG, Friedman DS. Glaucoma in China (and worldwide): changes in established thinking will decrease preventable blindness. Br J Ophthalmol. 2001; 85(11):1271-2.
3 Vithana EN, Khor CC, Qiao C, Nongpiur ME, George R, Chen LJ, et al. Genome-wide association analyses identify three new susceptibility loci for primary angle closure glaucoma. Nat Genet. 2012;44(10):1142-6.

4 Khor CC, Do T, Jia H, Nakano M, George R, Abu-Amero K, et al. Genome-wide association study identifies five new susceptibility loci for primary angle closure glaucoma. Nat Genet. 2016;48(5):556-62.
5 Nongpiur ME, Khor CC, Jia H, Cornes BK, Chen LJ, Qiao C, et al. ABCC5, a gene that influences the anterior chamber depth, is associated with primary angle closure glaucoma. PLoS Genet. 2014;10(3):e1004089.

6 Awadalla MS, Thapa SS, Hewitt AW, Burdon KP, Craig JE. Association of genetic variants with primary angle closure glaucoma in two different populations. PLoS One. 2013 Jun 28; 8(6):e67903. 
7 Chen Y, Chen X, Wang L, Hughes G, Qian S, Sun X. Extended association study of PLEKHA7 and COL11A1 with primary angle closure glaucoma in a Han Chinese population. Invest Ophthalmol Vis Sci. 2014;55(6):3797802.

8 Tan C, Huang L, Yang Z. [Association of PLEKHA7, COL11A1 and PCMTD1-ST18 gene polymorphisms with primary angle closure glaucoma in ethnic Han Chinese from Sichuan]. Zhonghua Yi Xue Yi Chuan Xue Za Zhi. 2016;33(4):545-49. Chinese.

9 Day AC, Luben R, Khawaja AP, Low S, Hayat S, Dalzell N, et al. Genotype-phenotype analysis of SNPs associated with primary angle closure glaucoma (rs1015213, rs3753841, and rs11024102) and ocular biometry in the EPIC-Norfolk Eye Study. Br J Ophthalmol. 2013;97(6):704-7.

10 Shi H, Zhu R, Hu N, Shi J, Zhang J, Jiang L, et al. An extensive replication study on three new susceptibility loci of primary angle closure glaucoma in Han Chinese: Jiangsu Eye Study. J Ophthalmol. 2013;2013:641596.
11 Nongpiur ME, Wei X, Xu L, Perera SA, Wu $\mathrm{RY}$, Zheng Y, et al. Lack of association between primary angle-closure glaucoma susceptibility loci and the ocular biometric parameters anterior chamber depth and axial length. Invest Ophthalmol Vis Sci. 2013; 54(8):5824-8.

12 Zhuang W, Wang S, Hao J, Xu M, Chi H, Piao $\mathrm{S}$, et al. Genotype-ocular biometry correlation analysis of eight primary angle closure glaucoma susceptibility loci in a cohort from Northern China. PLoS One. 2018;13(11): e0206935.13.

13 Wang S, Zhuang W, Ma J, Xu M, Piao S, Hao J, et al. Association of genes implicated in primary angle-closure glaucoma and the ocular biometric parameters of anterior chamber depth and axial length in a northern Chinese population. BMC Ophthalmol. 2018;18(1):271.

14 Jedlitschky G, Burchell B, Keppler D. The multidrug resistance protein 5 functions as an ATP-dependent export pump for cyclic nucleotides. J Biol Chem. 2000;275(39):30069-74.

15 Wijnholds J, Mol CA, van Deemter L, de Haas M, Scheffer GL, Baas F, et al. Multidrug-resistance protein 5 is a multispecific organic anion transporter able to transport nucleotide analogs. Proc Natl Acad Sci USA. 2000; 97(13):7476-81.
16 Karla PK, Quinn TL, Herndon BL, Thomas P, Pal D, Mitra A. Expression of multidrug resistance associated protein 5 (MRP5) on cornea and its role in drug efflux. J Ocul Pharmacol Ther. 2009;25(2):121-32.

17 Stojic J, Stöhr H, Weber BH. Three novel ABCC 5 splice variants in human retina and their role as regulators of $\mathrm{ABCC} 5$ gene expression. BMC Mol Biol. 2007;8:42.

18 Tang FY, Ma L, Tam POS, Pang CP, Tham CCY, Chen LJ. Genetic association of the PARL-ABCC5-HTR3D-HTR3C locus with primary angle-closure glaucoma in Chinese. Invest Ophthalmol Vis Sci. 2017 Aug 1; 58(10):4384-9.

19 Zeger SL, Liang KY, Albert PS. Models for longitudinal data: a generalized estimating equation approach. Biometrics. 1988;44(4): 1049-60.

20 Hanley JA, Negassa A, Edwardes MD, Forrester JE. Statistical analysis of correlated data using generalized estimating equations: an orientation. Am J Epidemiol. 2003;157(4): 364-75.

21 Rice TK, Schork NJ, Rao DC. Methods for handling multiple testing. Adv Genet. 2008; 60:293-308. 